\title{
Object Discovery and Acknowledgment in Images
}

\author{
${ }^{1}$ Jai Kishan Karwasra, ${ }^{2}$ Dr. Pankaj Dadhich \\ ${ }^{1}$ Ph.D. Scholar, Dept. of Computer Science, Tantia University, SriGanganagar(Raj.), India \\ ${ }^{2}$ Assistant Professor(C.S.), Dept of Computer Science, Tantia University, SriGanganagar(Raj.), India
}

\begin{abstract}
Object Acknowledgment is an innovation in the field of PC vision. It is viewed as one of the troublesome and testing errands in PC vision. Numerous methodologies have been proposed before, and a model with another methodology which isn't just quick yet additionally dependable. Easynet model has been contrasted and different models also. Easynet model takes a gander at the entire picture at test time so its forecasts are educated by worldwide setting.At the expectation time, our model produces scores for the nearness of the item in a specific class. It makes expectations with a Single system assessment. Here Object discovery is a relapse issue to spatially isolated jumping boxes and related class probabilities.
\end{abstract} $* * * * *$

\section{INTRODUCTION}

All Object Acknowledgment has two sections Category Acknowledgment and its identification. Category Dicovery manages recognizing the item from the foundation. Also, Category Acknowledgment manages characterizing the Object into one of the predefined classes. It is a recognizing procedure of explicit item in an advanced picture or video. For the most part, Object acknowledgment calculations depend on coordinating, learning, or example acknowledgment calculations utilizing appearancebased or highlight based systems. For instance, it is utilized to discover occasions of genuine articles like bikes, organic products, creatures and structures in pictures or recordings.

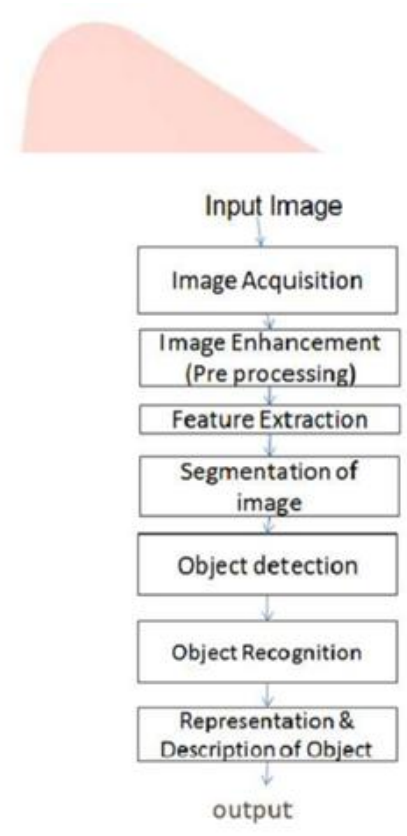

Fig 1: Model Diagram
As appeared in fig1. the Object Acknowledgment calculations use highlights which can be separated to perceive a specific Object. This model is straightforward and simple to execute. Here, object identification is a solitary relapse issue which recognizes legitimately from bouncing box arranges and class likelihood. Each Object has its own class, for example, all circles are round, which are utilized while perceiving the items.

\section{PREPROCESSING}

It is the most reduced degree of reflection. The procedure of preprocessing improves the picture force by stifling the undesirable highlights or upgrading them for additional processing. It resizes the picture size to $448 * 448$ and furthermore standardizes the differentiation and brilliance impacts. The picture is additionally trimmed and resized so highlight extraction can be performed effectively. The information pictures are pre-handled and effectively standardize the complexities and brilliance. Preprocessing step should be possible by subtracting the mean of picture forces and gap by the standard deviation. New splendor worth can be found by utilizing the area of a pixel in the info picture. The fig 2 underneath shows the preprocessing of picture.

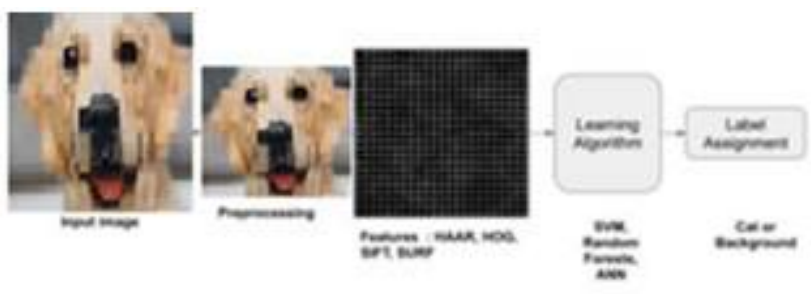

Fig 2. Preprocessing ( The image has been resized) 


\section{FEATURE EXTRACTION}

Its primary thought process is to rearrange the picture by considering just the significant data and forgetting about the additional data which isn't essential for acknowledgment. It utilizes the technique for edge discovery which can just hold the basic data. It speaks to the diminished piece of a picture as an element vector. This methodology is utilized when the size of picture is exceptionally enormous. Consequently, by this procedure, picture acknowledgment gets simpler. It begins from the effectively estimated information and highlights which gives some sort of data encouraging the further advances.

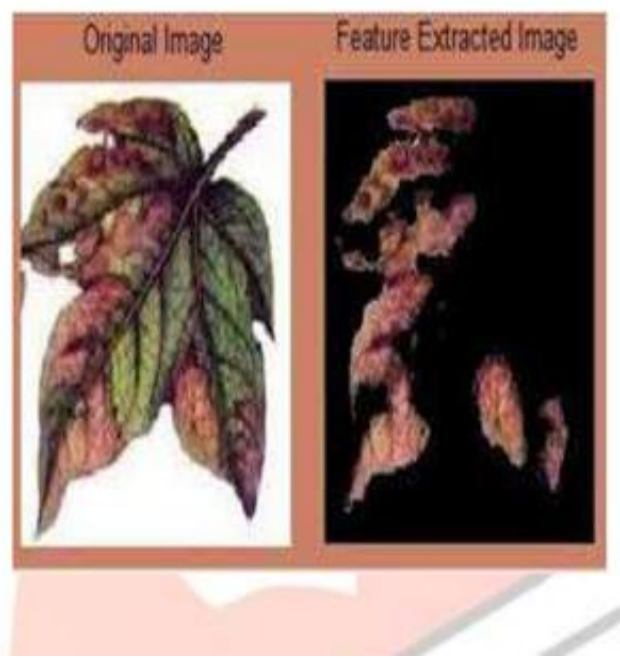

Fig 3: Feature Extraction (The image has been simplified considering only the important information).

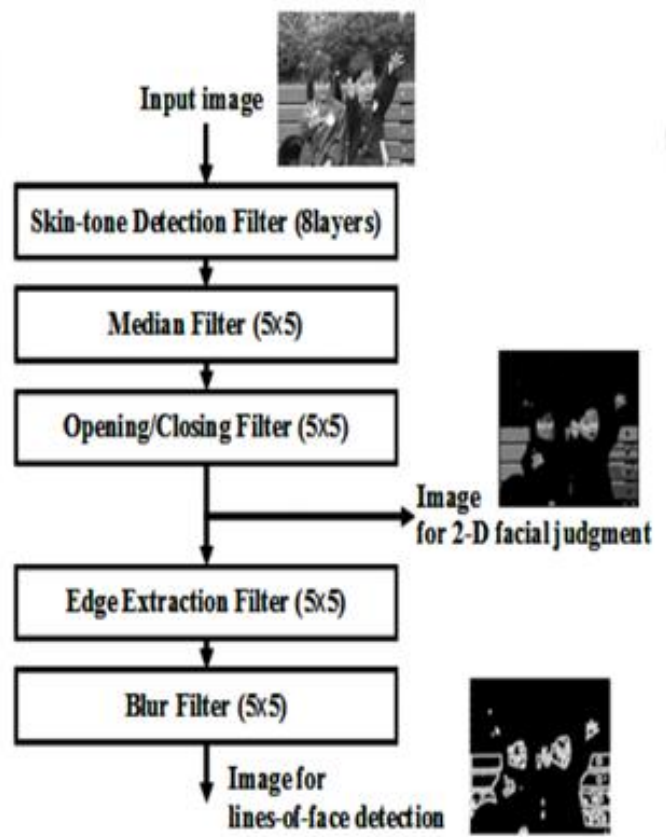

Fig 4: Combined process of Preprocessing and feature extraction.

\section{DICOVERY IN IMAGES}

The various segments of item identification are coordinated into a solitary neural system, which uses highlights from the entire picture to anticipate a jumping box. The bouncing boxes for different classes are likewise anticipated at the equivalent time. Hence the neural system investigations the full picture and furthermore the various articles in the picture.

The picture is contribution to the framework which is isolated into a matrix of $\mathrm{SxS}$ cells. In the event that the focal point of our picture falls in a framework cell, it is liable for examining that item. A matrix cell predicts $\mathrm{B}$ bouncing boxes. A jumping box is a square shape encasing an item. Each crate has a certainty score relating to it, which shows a rate demonstrating the degree to which it is sure that the container really encases some Object. This score doesn't reveal to us anything about the idea of the Object in the crate. In the event that no Object exists in a cell, the certainty score is zero. For each bouncing box, the cell additionally predicts a class from all the potential classes of our dataset. The certainty score for a container and class expectation are joined into a solitary score that disclose to us the likelihood that this specific jumping box contains a particular sort of item. Each Bounding box has 5 parameters: x, y, w, h, and certainty. The $\mathrm{x}$ and $\mathrm{y}$ organizes speak to the focal point of the bouncing box. The width (w) and tallness (h) are anticipated for the picture and the certainty score is additionally anticipated. For the PASCAL VOC dataset, a $7 \times 7$ lattice is utilized for example $S=7$ and 2 jumping boxes for every cell for example $\mathrm{B}=2$. As the PASCAL VOC has 20 classes so $C=20$. Subsequently, our last expectation is $7 \times 7 \times 30$ tensor. As there are $7 \times 7=49$ lattice cells and 2 jumping boxes for every cell, the all out number of bouncing boxes ends up being 98 . The greater part of these have low certainty scores and are in this manner disposed of.

\section{DESIGN}

A convolutional neural system is utilized for our model. A convolutional neural system is like a normal neural system and contains neurons and loads for every neuron. While a customary neural net doesn't scale well to accept full pictures as information, a 
convolutional neural net can accept enormous pictures as information and their engineering is structured in like manner. Three primary layers are utilized for a convolutional neural net: Convolutional Layer, Pooling Layer, and Fully-Connected Layer. The underlying layers of the neural net are utilized for highlight extraction while the completely associated layers foresee the directions and yield probabilities. This system has 24 introductory convolutional layers and 2 completely associated layers.

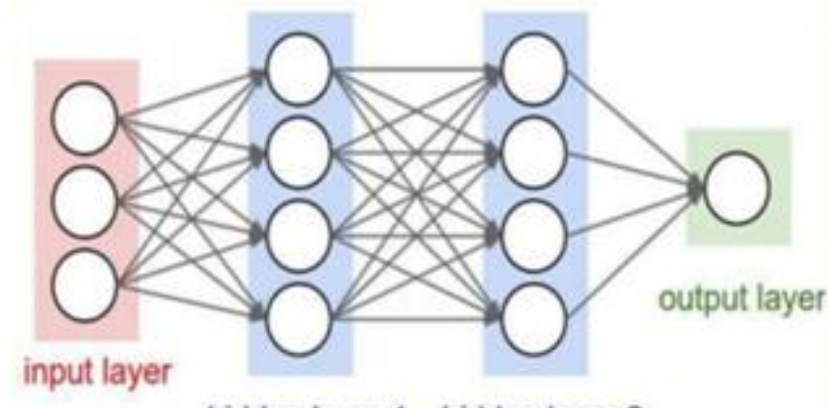

hidden layer 1 hidden layer 2

Fig 5 : Convolution neural network.

At last, an info picture (resized to $416 \times 416$ pixels) is passed to the convolutional neural net in a solitary pass, which turns out as a $7 \times 7 \times 30$ tensor, portraying the jumping boxes for matrix cells. The last scores for the bouncing boxes are determined and the ones having low scores are disposed of.

\section{DATASET}

The PASCAL VOC (visual Object classes) 2007 is a dataset which contains 9,963 pictures having a place with 20 distinct classes. The classes are referenced beneath -

\section{- $\quad$ Person: individual.}

- Vehicles: bike, motorbike, transport, vehicle, train, pontoon, aero plane.

- Animals: winged creature, feline, hound, bovine, sheep, horse.

- Indoor objects: bottle, television/screen, seat, eating table, couch, pruned plant.

Here is an example picture which gives one item from every one of the 20 distinct classes -

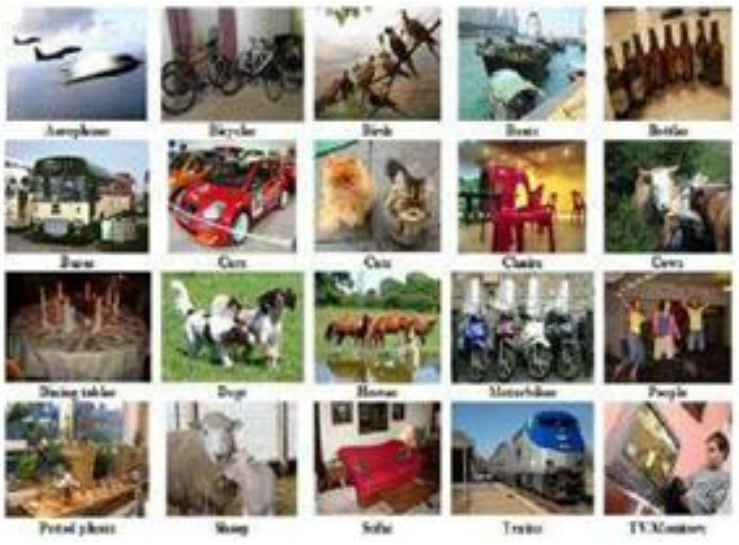

Fig 6: Dataset PASCAL VOC 2007.

\section{CHALLENGES FACED IN OBJECT ACKNOWLEDGMENT}

Change in size, trimming out the foundation are a portion of the components affecting the precision of the framework. The exactness of the model may change by scaling the picture.

Modifying Brightness and Contrast of the picture may likewise make it hard for the framework to perceive the articles in the picture. There might be situations when the item probably won't be noticeable enough for the framework to remember it. The Object Acknowledgment System must deal with these instances of low perceivability.

The framework may bomb in situations where comparative articles happen in gatherings and are excessively little in size. Different lightning conditions and shadows in the picture may likewise present trouble for the framework to perceive the object.

\section{APPLICATIONS OF OBJECT DICOVERY AND ACKNOWLEDGMENT}

1. Self-Driving Cars-Self Driving Cars may utilize Object discovery and acknowledgment framework to distinguish walkers and vehicles on the streets and afterward settle on the appropriate choice in understanding.

2. Face Dicovery-Another use of Object location and acknowledgment is Face Dicovery .e.g.- Facebook perceives individuals before they are labeled in pictures. 
3. Medical Science-Object Dicovery and acknowledgment framework may assist Medical science with detecting sicknesses. For e.g.- Detecting Tumors and different malignant growths.

4. Text Acknowledgment-Text acknowledgment manages perceiving letters/images, singular words and arrangement of words. Ex-Recognizing penmanship of an individual.

5. Hand Gesture Acknowledgment-Hand Gesture Acknowledgment manages acknowledgment of hand postures, and communications through signing.

\section{COMPARISON WITH OTHER DICOVERY SYSTEMS}

This model has been contrasted and other Dicovery Systems, for example, RCNN (Region based Convolution Neural Network), FASTER RCNN, SDD (Single Shot Detector) utilizing PASCAL VOC 2007 dataset.

1. RCNN-RCNN utilizes Selective Search to make the Bounding Boxes. RCNN takes a gander at the picture through windows of various sizes [9]. It removes the area proposition and afterward pass them however the CNN to create CNN highlights. Finally it includes SVM(support vector machine) which helps in arranging whether there is an Object in the district proposed ,and on the off chance that indeed, at that point what object it is .RCNN delivers around 18001900 bouncing boxes, while our framework creates just around 100 which is far not as much as that created in RCNN.

FASTER RCNN-FASTER RCNN is like RCNN with the exception of that it utilizes ROIPOOL (Region of intrigue Pooling).It runs the CNN just once per picture and offers its calculation to other sub locales. Quicker RCNN in this manner utilizes just one go of the first picture.

It can likewise be utilized for area proposition. It has mAP of around 70 .

Its downside is constant execution, which this model survives.
X. EVALUATION METRICS

\begin{tabular}{|c|c|c|c|}
\hline Method & mAP & FPS & $\begin{array}{c}\text { Batch } \\
\text { Size }\end{array}$ \\
\hline RCNN & 66.1 & 6 & 1 \\
\hline $\begin{array}{c}\text { Faster } \\
\text { RCNN }\end{array}$ & 73.2 & 7 & 1 \\
\hline $\begin{array}{c}\text { EASYNET } \\
\text { MODEL }\end{array}$ & 69.4 & 45 & 1 \\
\hline
\end{tabular}

Fig 7 : Evaluation metrics (Comparison of Easynet with RCNN and Faster RCNN)

$$
\operatorname{MAP}=\frac{\sum_{q=1}^{Q} A \text { veP(q) }}{Q}
$$

Mean average Precision (MAP) for a set of queries is the Mean of the average precision scores for each query, where $\mathrm{Q}$ is the number of Queries.

\section{OUTPUTS}

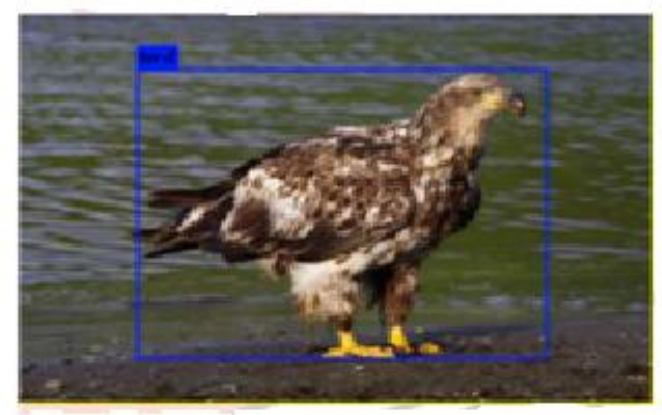

Fig 8(a) : Output of image eagle.jpg. OUTPUT : Bird ACCURACY : $91 \%$

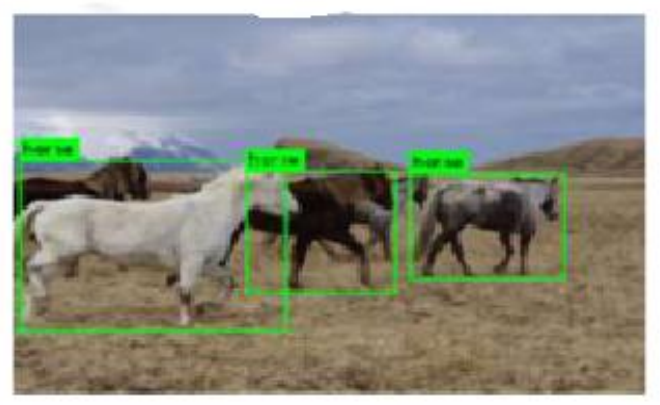

Fig 8(b): Output of image horse.jpg OUTPUT : Horses ACCURACY : $86 \%$ 


\section{CONCLUSION}

This model has been contrasted and other Dicovery Systems, for example, RCNN (Region based Convolution Neural Network), FASTER RCNN, SDD (Single Shot Detector) utilizing PASCAL VOC 2007 dataset.

Easynet model is exceptionally easy to execute and construct. It is brought together for object Acknowledgment. It sums up the areas and can be prepared effectively on full pictures. It can likewise comprise of item following alongside location. Likewise, getting the related Dataset PASCAL VOC 2007 made the work simpler and thus the model could be actualized on equipment without any interferences. Various kinds of ID should be possible and numerous items can be distinguished by Easynet model. In object identification, foundation subtracting approach has been utilized when a picture is taken from a solitary camera with a static foundation. In future, the work can be reached out by distinguishing the moving articles with non-static foundation.

\section{REFERENCES}

[1] Redmon, Joseph, et al. "You only look once: Unified, real-time object detection." Proceedings of the IEEE Conference on Computer Vision and Pattern Recognition.2016.

[2] Bazeille, Stephane, et al. "Automatic underwater image pre-processing." CMM'06. 2006.

[3] Lazebnik, Svetlana, Cordelia Schmid, and Jean Ponce. "Beyond bags of features: Spatial pyramid matching for recognizing natural scene categories." Computer vision and pattern recognition, 2006 IEEE computer society conference on. Vol. 2. IEEE, 2006.

[4] Yeo, Boon-Lock, and Bede Liu. "Rapid scene analysis on compressed video." IEEE Transactions on circuits and systems for video technology 5.6 (1995): 533-544.

[5] Belongie, Serge, Jitendra Malik, and Jan Puzicha. "Shape matching and object recognition using shape contexts." IEEE transactions on pattern analysis and machine intelligence 24.4 (2002): 509-522.

[6] [6]LeCun, Yann, Fu Jie Huang, and Leon Bottou. "Learning methods for generic object recognition with invariance to pose and lighting." Computer Vision and Pattern Recognition, 2004. CVPR 2004. Proceedings of the 2004 IEEE Computer Society Conference on. Vol. 2. IEEE, 2004.
[7] Hinton, Geoffrey E., et al. "Improving neural networks by preventing co-adaptation of feature detectors." arXiv preprint arXiv:1207.0580 (2012).

[8] Krizhevsky, Alex, Ilya Sutskever, and Geoffrey E. Hinton. "Imagenet classification with deep convolutional neural networks." Advances in neural information processing systems. 2012.

[9] Girshick, Ross, et al. "Rich feature hierarchies for accurate object detection and semantic segmentation." Proceedings of the IEEE conference on computer vision and pattern recognition. 2014

[10] Ren, Shaoqing, et al. "Faster R-CNN: Towards real-time object detection with region proposal networks." Advances in neural information processing systems. 2015.

[11] Khan, Fahad Shahbaz, et al. "Color attributes for object detection." Computer Vision and Pattern Recognition (CVPR), 2012 IEEE Conference on. IEEE, 2012 